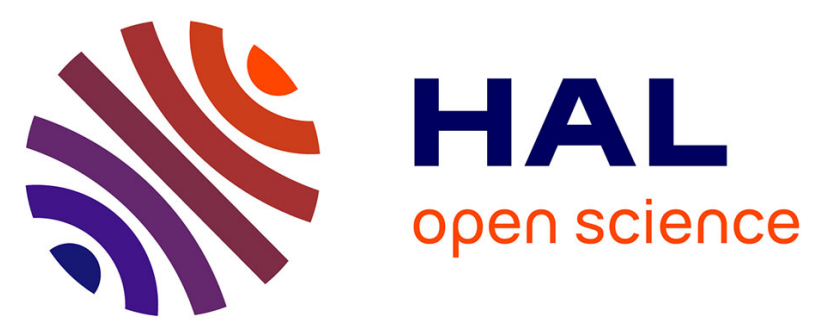

\title{
Even when Icons are Not Worth a Thousand Words They are Helpful in Designing Asthma mHealth Tools
}

\author{
Michael Lefco, Jensi Gise, Burton Lesnick, Rosa I. Arriaga
}

\section{To cite this version:}

Michael Lefco, Jensi Gise, Burton Lesnick, Rosa I. Arriaga. Even when Icons are Not Worth a Thousand Words They are Helpful in Designing Asthma mHealth Tools. 16th IFIP Conference on Human-Computer Interaction (INTERACT), Sep 2017, Bombay, India. pp.23-33, 10.1007/978-3-31967687-6_2. hal-01717194

\author{
HAL Id: hal-01717194 \\ https://hal.inria.fr/hal-01717194
}

Submitted on 26 Feb 2018

HAL is a multi-disciplinary open access archive for the deposit and dissemination of scientific research documents, whether they are published or not. The documents may come from teaching and research institutions in France or abroad, or from public or private research centers.
L'archive ouverte pluridisciplinaire HAL, est destinée au dépôt et à la diffusion de documents scientifiques de niveau recherche, publiés ou non, émanant des établissements d'enseignement et de recherche français ou étrangers, des laboratoires publics ou privés. 


\title{
Even when Icons are Not Worth a Thousand Words They are Helpful in Designing Asthma mHealth Tools
}

\author{
Michael Lefco ${ }^{1}$, Jensi Gise ${ }^{2}$, Burton Lesnick ${ }^{3}$, Rosa I. Arriaga ${ }^{1 *}$ (0000-0002-8642-7245) \\ ${ }^{1}$ Georgia Institute of Technology \\ Atlanta, GA, USA \\ *arriaga@cc.gatech.edu \\ ${ }^{2}$ Georgia State University \\ Atlanta, GA, USA \\ ${ }^{3}$ Children's Healthcare of Atlanta \\ Atlanta, GA, USA
}

\begin{abstract}
Asthma is the most common childhood chronic illness. Its management requires that caregivers have access to a variety of information. For example, asthma action plans (AAP) are written instructions for responding to escalating asthma symptoms. Icons have been used to facilitate the accessibility of health information and we investigate whether they benefit parents of young children. We conducted a 4-part study with 36 participants where we 1) gathered requirements for an asthma management mHealth tool 2) assessed the comprehension of icons to be used in an AAP 3) compared the usability of a text-based (T-B) vs. icon-based (I-B) AAPs 4) and gathered feedback about an mHealth tool we designed. Results suggest that the icons we developed were highly matched to their meaning ( $92 \%$ translucency). Usability findings showed that in general both AAPs were equally matched. However, benefits accrued to I-BAAP users in relation to greater accuracy and speed of response in scenarios that required parents to identify asthma symptoms. Twothirds of parents indicated that they would prefer to use the I-BAAP to manage their child's asthma. The remaining third preferred the T-BAAP because it contained more details. We conclude with recommendation on how designers and researchers can improve mHealth tools for caregivers of asthmatic children.
\end{abstract}

Keywords: Asthma; Asthma Action Plan; Icon-Based Asthma Action Plan; Asthma App; Mobile Application; Mobile Computing; mHealth; Application

\section{Introduction}

Asthma is a chronic respiratory condition. It is the most common disease among children with a $14 \%$ prevalence worldwide [32]. When not properly managed, asthma can lead to lessened quality of life and increased avoidable emergency department (ED) visits, hospitalizations, and deaths [30]. Asthma is a public health problem in both wealthy and developing nations [32]. Requirements for appropriately managing asthma are well known [6, 11]. Additionally, the Global Asthma Report indicates that health authorities in all countries should ensure that all patients are provided with a written asthma action plan (AAP) [6].

An AAP is a health management tool given to patients by their medical provider. It illustrates what actions to take at different levels of symptom severity from day-to-day (i.e., Green Zone) medication use to emergency situations (i.e., Red Zone). AAPs are important in enhancing the patient's or caregiver's understanding of the disease, encouraging self-management, [29] and 
creating a partnership between the physician and caregiver. Research suggests that this partnership coupled with strong communication between caregivers and physicians can lead to better medication adherence [1]. Furthermore, research has shown that children with written AAPs compared to those without AAPs are half as likely to be hospitalized or visit the emergency department [24].

Currently, AAPs given to children and caregivers are text-based paper documents. Studies have found that including icons and text (as compared to text alone) on documents may increase the likelihood that individuals being discharged from emergency rooms will read the information, do what was recommended [10] and retain the knowledge [4]. Houts et al. found that for the general public, pictures that are closely linked to text increase attention to and recall of health information better than text alone. They also found that pictures enhance adherence to health instructions and target behaviors for individuals with lower literacy [15]. These findings suggest that designers can positively impact health management by designing tools that include icons, pictures, and images in medical information.

Healthcare providers can encourage use of the AAP by reviewing it with the patient at each doctor visit and highlighting successful use as a way to control asthma symptoms. Ideally, selfmanagement approaches should be tailored to the needs and literacy levels of patients and asthma management tools should be accessible at all points of care, from the hospital to the home $[11,14]$. Usability and accessibility of an AAP are very important for the overall health of children with asthma. However, while 1 in 11 children in the United States have asthma, less than 1 in 2 children get an AAP [6]. Not having an AAP or not having access to one (e.g., because a parent did not send it to school) is a significant barrier to effective health management.

Our research has two goals. First, to investigate if using icons can help facilitate the asthma management of children with asthma. We addressed this goal by designing an icon-based asthma action plan (I-BAAP) using information from interviews with caregivers of children with asthma. Then we assessed mom's comprehension of asthma icons and finally we tested them on various scenarios using either an I-BAAP or a text based (T-B) AAP for correctness and speed of user response. This allowed us to assess any benefits of the I-BAAP over the TBAAP for speed and accuracy of mom's response to common asthma symptoms. Our second goal is to investigate caregivers' receptiveness to using a mobile asthma management tool. We addressed this goal by designing a mobile I-BAAP and then gathering qualitative information from caregivers on its pros, cons, and usability. With our findings, we hope to bridge gaps in the literature regarding how icons, compared to text, can be of value for parents of children with asthma, and to better understand how mobile technology designers can improve usability of AAPs.

\section{$2 \quad$ Related Works}

There is a long history of studying the use of icons in HCI [13, 31] and health management [4]. There are few studies about the use of icons in asthma management tools. Adults have been the sole focus of research that has been done to study how images impact asthma management. Yin, et al. found that when physicians used an AAP with pictorial descriptions to provide asthma counseling in a patient-doctor scenario, these physicians provided clearer counseling than physicians who used a standard text-based AAP. The delivery of clearer communication and deeper coverage of content from physicians is believed to have a positive impact on asthma management [32]. Researchers have sought to leverage the positive outcomes found by including icons in health management material for adults with asthma. Roberts et al. examined benefits of a pictorial-based AAP among three different populations in the United Kingdom. 
The pictures used were well understood and the pictorial-based AAP was well received by participants. In this study, the adults with asthma were able to adequately recount appropriate actions to take in clinical scenarios using the pictorial-based AAP [26]. While this study shows the benefit of icons for physicians and adults with asthma, little research exists on how an iconbased, rather than text-based, AAP may help caregivers of young children with asthma deliver effective asthma care.

Asthma is a vibrant area of research in the mobile health (mHealth) space. This has run the gamut from design [20,23], web-based technologies [3], instruments, [22] to interventions [3436]. Huckvale and colleagues have conducted a number of studies to investigate mobile asthma management tools [16-18]. In reviewing over 100 asthma apps they found that apps were not likely to provide information for self-care based on evidence in medicine, or focus on evidencebased interventions such as the use of an asthma action plan. However, a pilot study has found that adults with asthma perceived that a mobile application of a text-based asthma action plan could improve their ability to control their asthma [23].

Our study seeks to bridge three gaps in the literature review. First, rather than focus on adults with asthma we focus on the mothers of young children with asthma. Specifically, we investigate caregiver's use of an icon-based asthma action plan in asthma management scenarios for their young child. Second, while studies have investigated the benefit of iconbased AAPs, there is no study that actually compares them against traditional text-based AAPs. Third, while previous studies have focused on adults' perception of asthma action plan mobile applications, we will investigate mothers' attitudes toward using this technology to care for their child with asthma.

\section{$3 \quad$ Methods and Results}

We conducted a 4-part study with 36 total participants where we 1) gathered requirements for an asthma management mHealth tool 2) assessed the comprehension of icons to be used in an AAP 3) compared the usability of a text-based (T-B) vs. icon-based (I-B) AAPs 4) and gathered feedback about the mHealth tool we designed. Of the 36 participants, five participated in the first part of the study, 7 participated in the 2nd part of the study, and the remaining 24 participated in the 3rd, and 4th parts of the study. All participants provided informed consent before joining the study.

In part 1, we conducted interviews with 5 individuals including a pulmonologist (1), an asthma educator (1), nurse practitioners (2), and a parent (1) to design a mHealth tool. Interviews were focused on how medical professionals provide information and how parents receive it as well as important factors in asthma health management. The interviews were semi-structured to allow participants to drive the conversation towards the most important issues. Audio from the sessions was recorded. The interviews were professionally transcribed. Then 2 reviewers read through the transcription looking for design implications for the mobile I-BAAP. The 2 reviewers compared their notes to jointly confirm design ideas for the application. We used the gold standard of care for US, the National Heart, Lung, and Blood Institute's (NHLBI) asthma action plans (see Fig. 1A) to develop our paper-based I-BAAP. We designed our I-BAAP in paper-format because paper-based asthma action plans are the most common forms and it allowed us to test it against traditional text-based asthma action plans. A member of the research team designed the I-BAAP. He studied existing action plans and health iconography and used his design expertise in creating the paper I-BAAP (Fig. 1B). It is important to note that our goal with creating an asthma management tool was to develop a tool that included an AAP and other features to support parents. A prototype for the mobile health tool was built using the Twitter Bootstrap web framework (Fig 1C). 
Interview analyses led to the inclusion of a number of features for the mobile application beyond the I-BAAP. Next, we provide a number of examples. The certified asthma educator recommended including notifications about asthma related events and reminders for medication refills and doctor appointments. Nurse practitioners advised that we distinguish between green and yellow zone medications, illustrate pre-treating before exercise, show alternative names of medicines, and enable a calendar tracker. A pulmonologist suggested we display pictures of the asthma paraphernalia (e.g., the chamber and age related devices), and the mom we interviewed desired the ability to track symptom flare-ups in the app to be able to share with a doctor.

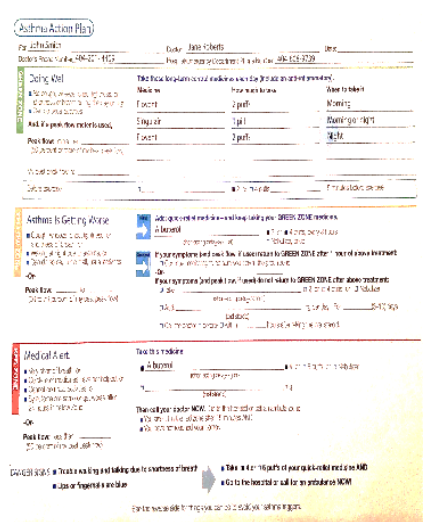

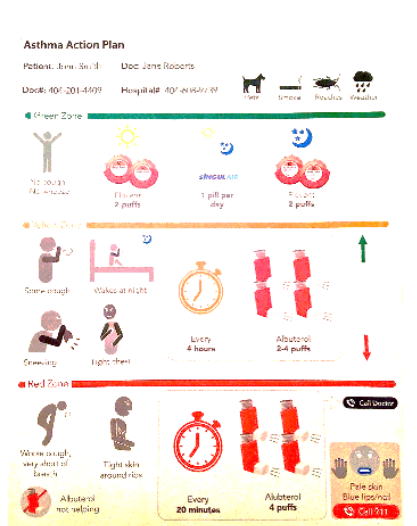

C

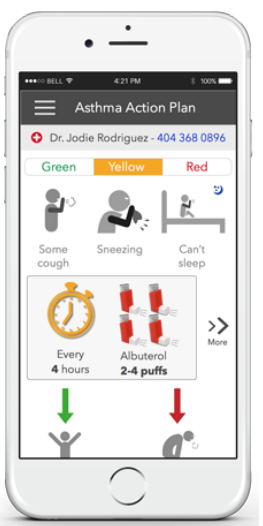

Fig. 1. Gold standard Text (A)-, Icon (B)-based asthma action plan and (C) mobile tool

Only moms of children with asthma were present at the clinic where we recruited participants for this study. For the rest of the paper we refer to them interchangeably as "moms" or "participants." Thirty-one participants were recruited for Part 2-4 of the study in the waiting room of a local pulmonology clinic. Seven participated in part 2 of the study and the remaining 24 participated in parts 3 and 4 of the study. Since they all filled out the same survey we discuss those results first. We note, however, that some moms opted to forgo answering certain questions. Thus, there is not a complete set of answers for all items on the survey. On the survey we asked about demographic information, technology adoption, and questions related to the child's asthma management. Participants had at least 1 child with asthma (though some had multiple children with asthma, mean $=1.5$ ). They were asked to report information for the child who was with them at the clinic. Participants had varying educational backgrounds ranging from leaving school in the $8^{\text {th }}$ grade to a master's degree. Of the participants, 17 reported having public insurance, 7 had private, 4 had none, and 2 did not respond. Twenty-five participants were African American, 3 White, 1 Hispanic, and 1 did not respond. Demographics of the sample are representative of the moms and patients in the clinic on the days we recruited and conducted interviews.

Information on the participant's use of technology revealed 27/31 moms owned a smartphone and 23 surfed the Internet on their phone more than once per day. Nineteen/31 moms reported that they had not used technology to help manage their child's asthma. Mom's reported that they were very confident about their ability to control their child's asthma and their ability to provide a clear picture of their child's health to their doctor (avg. $4.41($ S.D. $=1.10)$ and 4.53 $($ S.D. $=1.08)$, respectively). These two survey items were on 5-point scale .

Survey responses revealed that the mean age of the child that was with them at the clinic was 8.25 years. Moms were asked to use the NHLBI severity index to indicate their child's asthma 
status. The severity level of the asthmatic children varied; 11 were reported as having severe asthma, 11 moderate, 4 low, and 1 did not respond. Twenty-six of $31 \mathrm{moms}$ had an asthma action plan that they received in the last year and the rest did not remember getting one. Only 6 participants reported having the AAP with them (in their purse, child's book bag, etc.). Twentyone/26 participants reported having shared their AAP with friends, family, or the child's school. In Part 2 we tested the comprehension of the icons (with $7 \mathrm{moms}$ ). This was done using a paper evaluation in which users were presented with the icons (see in Fig. 1B and 1C) and asked to rate how well the icon matched our intended definition (translucency). Average translucency ratings for our icons were very high at $92 \%$, or on average greater than 6.4 out of 7 , and indicated that intended meanings match across the 11 icons. While the sample for testing icon comprehension was small, we found high agreement among the participants; because these results were similar to previous findings [26] we proceeded to the next phase of the study.

In Part 3, we investigated if icons could enhance participants' ability to manage their child's asthma symptoms. We did this by comparing text-based asthma action plans (T-BAAP, Fig. 1A) to icon-based asthma action plans (I-BAAP, Fig. 1B) using a scenario assessment test used in previous research [26]. We recruited 24 females who were randomly assigned to one of two groups (I-BAAP or T-BAAP). They were asked to use their AAP to explain how they would respond to 5 specific asthma-care scenarios [26], (see Table 2). The researcher recorded participants' responses and time taken to give the response. After each scenario, participants were asked about their confidence in answering the question and the ease of use of the AAP. At the end of the scenarios test, participants were shown the I-BAAP and the T-BAAP side by side and asked which they preferred to manage their child's asthma.

Table 2. Scenario Questions and Percentage Correct (* indicates statistically different values for accuracy and ${ }^{\times}$indicates statistically faster responses for correct answers)

\begin{tabular}{|c|l|c|c|}
\hline Q1 & $\begin{array}{l}\text { N=12 in each group } \\
\text { Your child wakes up during the night coughing or } \\
\text { wheezing. What actions should you take? }\end{array}$ & $58 \%$ & $*^{\times} 92 \%$ \\
\hline Q2 & $\begin{array}{l}\text { Are there any medications that your child should } \\
\text { take every day? If so, what are they and when } \\
\text { should they be taken? }\end{array}$ & $82 \%$ & $83 \%$ \\
\hline Q3 & $\begin{array}{l}\text { When should you contact your doctor? } \\
\text { Q4 }\end{array} \begin{array}{l}\text { Your child has had a cold for several days so you } \\
\text { have started him on his quick relief medicine. You } \\
\text { notice that he is still coughing and that his fingernails } \\
\text { are a blue color. What action(s) should you take? }\end{array}$ & $63 \%$ \\
\hline Q5 & \begin{tabular}{l} 
What is the name of the quick relief medicine? \\
\hline
\end{tabular} & $66 \%$ & $*^{\times} 75 \%$ \\
\hline
\end{tabular}

Results from part 3 indicated that confidence in response and ease of use was basically at ceiling for both asthma action plans ( $\sim 6.5 / 7$ across all scenarios). Two-tailed t-tests were conducted to compare performance between the two AAP groups. Results showed that the difference in average speed for correct responses was significantly faster for I-BAAP users $(\mathrm{M}=3.22, \mathrm{SD}=1.23)$ than for T-BAAP users $(\mathrm{M}=6.21, \mathrm{SD}=4.43)(\mathrm{t}(22)=2.1572, \mathrm{p}<0.05$, Cohen's d $=0.8807$ ).

The two AAPs led to similar accuracy rates in $3 / 5$ scenarios (see Table 2). However, there was a statistically significant difference between accuracy for scenarios 1 and 4 . Results from a 
post-scenario test question about I-BAAP versus T-BAAP preference also showed that $15 / 24$ participants thought that the I-BAAP would be more helpful in managing their child's asthma than the T-BAAP. All 24 moms were interested in "trying out" a mobile asthma health tool.

In part 4 moms in the waiting room of a local pulmonology clinic were presented with the mobile health (mHealth) tool we developed (Fig. 1C). The researcher instructed participants to explore the tool and to speak their thoughts as they interacted with it and to provide their general impressions of the tool. Each lasted approximately 10 minutes. Sessions were recorded for further analysis and feedback was organized into themes related to usability issues, reasons for use, understanding of asthma information and suggestions for improvement.

Part 4 data shows that there is general interest and receptiveness in using mHealth tools (all moms wanted to interact with it even if they did not have a smartphone). Part 4 also provided a set of recommendations for improvements to the mHealth tool. These included adding a medication alarm, the ability to personalize calendar information, the ability to send data in the application to their doctor as to give them a sense of how the child was doing before arriving for an appointment. These suggestions may all serve to improve the tool in future iterations. The biggest usability problems that emerged were that only $2 / 24$ moms spontaneously found the navigation menu (which includes links to the triggers page, notifications, and the send plan feature) and the medication tracking feature in the AAP's "green zone."

\section{Discussion}

Our research bridged three gaps in the literature that can inform future mobile health tool development. First, we focused on mothers of children with asthma rather than adults with asthma [26, 23] or medical professionals [32]. Second, we compared the effectiveness of iconbased (I-B) asthma action plans (AAPs) versus text based (T-B) AAPs in terms of accuracy and speed of response to common asthma symptoms. Previous work had focused on designing IBAAPs and testing their usability [15] but there had never been an exploration of the comparative strengths for managing asthma symptoms. Third, we gathered feedback for an mHealth tool for managing asthma that can serve to inform design and research with parents of young children.

In our study moms were presented with 5 different asthma management scenarios and provided either a T-BAAP or I-BAAP to answer questions related to these scenarios. We found that the I-BAAP had two main benefits over the T-BAAP. First, it facilitated identifying the relation between symptoms and medication. Images of figures that were coughing, wheezing, (scenario 1) or turning blue (scenario 2) allowed moms to easily identify the correct medicine to administer. Second, the I-BAAP led to significantly faster response times in 3 of the 5 scenarios and those faster speeds were associated with the correct response. Thus, moms that used the IBAAP compared to moms' with the traditional AAP responded to asthma management scenarios more quickly and more accurately. We also found that the mom with the lowest educational level and lowest confidence in her ability to manage her child's asthma benefitted most by the I-BAAP.

The asthma management test indicated a trend where participants who used the I-BAAP had slightly lower accuracy on the question that asked for the name of the quick relief medication. This resonates with other health-related findings [4, 10, 15] that pairing text and images is helpful. This suggests that in future iterations of an I-BAAP, the medication icons should include larger or clearer text to make the medication name more identifiable.

Our study differed from other research that showed that icons had a profound impact on processing health information $[4,15]$. This could be related to the fact that parents of children are more engaged in the management of their child's illness than adults are in their own health 
management. Support for this notion comes from that fact that moms in our study had high confidence in their ability to take care of their children and in their ability to communicate with the physicians. We also did not find that non-white participants benefited more from icon-based versus text-based health information [4]. This likely reflects that fact that education mediates the benefits of including icons in health information and our participants had high educational attainment.

Our study faces several limitations, which in turn provides ample opportunity for future research. While we did not intend to exclusively recruit females, however, only moms were present at the clinic where this study took place. There is no reason to believe that dads would have performed differently than moms but this is still an open question. Another limitation includes the small size and makeup of our sample population. Future studies should focus on participants with low literacy and low confidence in their ability to care for children with asthma (e.g., parents of newly diagnosed children or baby sitters or even the children themselves). Our results replicate findings that [26] I-BAAPs can be used to identify the correct actions to take when presented with asthma scenarios. However, there is no evidence that performance in the scenario test is related to asthma management in the real world. Future studies should deploy I-BAAP vs. T-BAAP and see if the advantages we noted (improved speed and accuracy) translate to dealing with asthma exacerbation at home or school.

Our study shows that while there may be a variety of mobile asthma tools [16-18] there is still much work for the HCI community in this space. For example, the technology utilization survey showed that while $66 \%$ of participants surf the web on their phone, only $10 \%$ use their phone to help manage their child's asthma. Likewise, $79 \%$ of the participants reported that their action plan was away from them, but $87 \%$ owned mobile phones. Thus, a mobile tool maybe a way to app could increase accessibility to the action plan. Our study shows that parents are open and receptive to mobile health tools that include both AAP and other features to manage their child's asthma and to share that information with other caregivers (teachers, other family members and doctors). These tools can also mediate communication between parents and doctors between scheduled visits. Previous research [33] showed that doctors communicate more clearly when using an I-BAAP than a T-BAAP. This maybe yet another reason to include I-BAAPS in the mHealth tool.

There are a number of implications from this study for both designers and researchers. One recommendation for icon designers is that they use Roberts et al.'s measure of translucency, how well icons matched their meaning [26]. This metric has now been successfully used across various patient populations in the UK and US. Our study also provides evidence that designers should include text to underscore important information in icon-based health tools. We found that some of the moms preferred the T-BAAP because it had more information than the IBAAP. Likewise, we found that the one scenario where moms had to identify the name of the medicine was slower for the I-BAAP ground (where there were no labels). A take-away for researchers is that it is important to include both subjective and objective measures of usability. For example, we found ceiling effects for subjective measures "ease of use" even for scenarios where objective data (accuracy and response time) showed that parents were not answering accurately. Thus, it is not enough to ask users if a design or prototype works but to have them show you that they can use it.

\section{Conclusion}

Our study informs future directions for mHealth research with parents of children with asthma. The fact that two-thirds of the participants preferred the I-BAAP over the T-BAAP for managing their child's asthma suggests that this is an area rich with opportunities. Our study 
suggests that I-BAAPs may be beneficial in allowing parent to more quickly and accurately react to their child's asthma symptoms. Also, that it is important to include text in icon-based informational material. However, these results are limited by the fact that they were not conducted in the "real world". Broader implications include the fact that it is important for researchers to include both subjective and objective measure of usability. Specifically we found that self-report measures of "confidence" and "ease of use" were at times uncorrelated with accuracy of response.

\section{Acknowledgments}

We thank all of the participants for taking the time to aid us with our research.

\section{$7 \quad$ References}

1. Adams, R.,Appleton, S., Wilson, D., \& Ruffin, R. (n.d.). Participatory Decision Making, Asthma Action Plans, and Use of Asthma Medication: A Population Survey. Journal of Asthma, 673-678.

2. Annor F., Bayakly A., Vajani M., Drenzek, C., Lopez F., O'Connor J., Yancey M. Georgia Asthma Prevalence Report. Georgia Department of Public Health, Health Protection, Epidemiology, Chronic Disease, Healthy Behaviors and Injury Epidemiology Section, December 2013.

3. Arguel, A., Lau, A., Dennis, S., Liaw, S., \& Coiera, E. (n.d.). An Internet Intervention to Improve Asthma Management: Rationale and Protocol of a Randomized Controlled Trial. JMIR Research Protocols, E28-E28.

4. Austin PE, Matlack R, Dunn KA, Kosler C, Brown CK. Discharge instructions: do illustrations help our patients understand them? Ann Emerg Med, 25(1995) pp.317-320

5. Cairns, P., \& Cox, A. L. (2008). Research methods for human-computer interaction. Cambridge, UK: Cambridge University Press.

6. CDC, National Center for Environmental Health, Division of Environmental Hazards and health Effects. Asthma's impact on the nation - Data from the CDC National Asthma Control Program. (2015). www.cdc.gov/asthma. Also, Global Asthma Report: http://ginasthma.org/wp-content/uploads/2016/04/GINA-2016-main-report_tracked.pdf

7. Chaudry, B., Connelly, K., Siek, K. \& Welch, J. (2012). Mobile Interface Design for LowLiteracy Populations. IHI'12, January 28-30, 2012. Copyright 2012 ACM.

8. Curtis, L., Wolf, M., Weiss, K., Grammer, L. (2012). The Impact of Health Literacy and Socioeconomic Status on Asthma Disparities. Journal of Asthma, 49:178-183.

9. D'Auria, J. (2013). All about asthma: Top resources for children, adolescents, and their families. Journal of Pediatric Health Care, Volume 27. Number 4.

10. Delp C., Jones J. (1996). Communicating information to patients: the use of cartoon illustrations to improve comprehension of instructions. Acad Emerg Med. 3:264-70.

11. Expert Panel Report 3 (EPR-3): Guidelines for the Diagnosis and Management of AsthmaSummary Report 2007. Journal of Allergy and Clinical Immunology. Volume 120. Number 5.

12. Gillette, C., Carpenter, D., Ayala, G., Williams, D., Davis, S., Tudor, G., ... Sleath, B. (2013). How Often Do Providers Discuss Asthma Action Plans With Children? Analysis of Transcripts of Medical Visits. Clinical Pediatrics, 1161-1167.

13. Gittins, D. 1986. Icon-based human-computer interaction. Int. J. Man-Machine Studies. 24, 
519-543.

14. Gupta, S., Wan, F., Hall, S., \& Straus, S. (n.d.). An Asthma Action Plan Created by Physician, Educator and Patient Online Collaboration with Usability and Visual Design Optimization. Respiration, 406-415.

15. Houts, P.S., Doak, C.C., Doak, L.G., Loscalzo, M.J. (2006). The role of pictures in improving health communication: A review of research on attention, comprehension, recall, and adherence. Patient Education and Counseling. 61, 173-190

16. Huckvale, K., Car, J. (2014). Implementation of mobile health tools. Journal of American Medical Association. Volume 311, Number 14.

17. Huckvale, K., Morrison, C., Ouyang, J., Ghaghda, A., Car, J. (2015). The evolution of mobile apps for asthma: an updated systematic assessment of content and tools. BMC Medicine. 13:58.

18. Huckvale, K., Car, M., Morrison, C., \& Car, J. (2012). Apps for asthma self-management: a systematic assessment of content and tools. BMC medicine. 144.

19. Jeong, H.Y. and Arriaga, R.I. "Using an ecological framework to design mobile technologies for pediatric asthma management," Proceedings of the 11th International Conference on Human-Computer Interaction with Mobile Devices and Services, 2009, p. 17

20. Jeong, H., Yun, T., Sung, J., Abowd, G., Arriaga, R., \& Hayes, G. (2011). Act collectively: Opportunities for technologies to support low-income children with asthma. Proceedings of HCI 2011 - 25th BCS Conference on Human Computer Interaction, 413-420.

21. Kascak, L., Rebola, C.B., Braunstein, R., Sanford, J.A. (2013). Icon Design to Improve Communication of Health Information to Older Adults. Communication Design Quartlerly 2.1,6-32.

22. Krisela Rivera, Nancy J. Cooke, and Jeff A. Bauhs. (1996). The effects of emotional icons on remote communication. In Conference Companion on Human Factors in Computing Systems (CHI '96), Michael J. Tauber (Ed.). ACM, New York, NY, USA, 99-100. Larson, E. Goel, M., Larson, E. Goel, Boriello, G. Heltshe, S., Rosenfeld, Patel S. Spirosmart using a microphone to measure lung function on a mobile phone. In Proceedings of the 2012 ACM Conference on Ubiquitous Computing (UbiComp '12). ACM, New York, NY, USA, 280-289. DOI=http://dx.doi.org/10.1145/2370216.2370261

23. Licskai C, Sands TW, Ferrone M (2013). Development and pilot testing of a mobile health solution for asthma self-management: asthma action-plan smartphone application pilot study. Can Respir J. 2013 Jul-Aug;20(4):301-6.

24. Lieu, T., Quesenberry, C., Capra, A., Sorel, M., Martin, K., Mendoza, G. Outpatient Management Practices Associated With Reduced Risk of Pediatric Asthma Hospitalization and Emergency Department Visits. Pediatrics. 1997:100(3).

25. Ring, N., Jepson, R., Hoskins, G., Wilson, C., Pinnock, H., Sheikh, A., \& Wyke, S. (n.d.). Understanding what helps or hinders asthma action plan use: A systematic review and synthesis of the qualitative literature. Patient Education and Counseling, E131-E143.

26. Roberts, N., Mohamed, Z., Wong, P., Johnson, M., Loh, L., \& Partridge, M. (n.d.). The development and comprehensibility of a pictorial asthma action plan. Patient Education and Counseling, 12-18.

27. Rudd, R., Kirsch, I., Yamamoto, K. Literacy and Health in America - Policy Information Report. Educational Testing Service. 2004. www.ets.org/research/pic

28. Saver, C. Overcoming low health literacy: Helping your patient understand. OR Manager. 2012:28(6)

29. Tan, N., Chuan, Z., Chen, Wern F. S., Heng Ngoh, A. \& Tai, B.C.. "Effects of a Written Asthma Action Plan on Caregivers' Management of Children with Asthma: A Crosssectional Questionnaire Survey." Primary Care Respiratory Journal 22.2 (2013): 188. Web. 
30. United States Environmental Protection Agency. (N.D.) Children's Environmental Health Disparities: Black and African American Children and Asthma. Retrieved September 22, 2015 from permanent.access.gpo.gov.

31. Worden, A., Walker, N., Bharat, K., and Hudson, S. 1997. Making computers easier for older adults to use: area cursors and sticky icons. In Proceedings of the ACM SIGCHI Conference on Human factors in computing systems (CHI '97).

32. World Health Organization statistics: http://www.who.int/respiratory/asthma/en/

33. Yin HS, Gupta RS, Tomopoulos S, et al. A Low- Literacy Asthma Action Plan to Improve Provider Asthma Counseling: A Randomized Study. Pediatrics. 2016;137(1):e20150468

34. Yun, T. J., Joeng, H. Y., Hill, T. D., Lesnick, B., Brown, R., Abowd, G. D., \& Arriaga, R. I. (2012). Using SMS to provide continuous assessment and improve health outcomes for children with asthma. In IHI'12 - Proceedings of the 2nd ACM SIGHIT International Health Informatics Symposium (pp. 621-630). DOI: 10.1145/2110363.2110432

35. Yun, TJ and Arriaga, R. I. A text message a day keeps the pulmonologist away. In Proceedings of the SIGCHI Conference on Human Factors in Computing Systems

(CHI '13). ACM, New York, NY, USA, 1769-1778. DOI: https://doi.org/10.1145/2470654.2466233

36. Yun, TJ and Arriaga, R. I. SMS is my BFF: Positive impact of a texting intervention on low-income children with asthma. In Proceedings of the 10th EAI International Conference on Pervasive Computing Technologies for Healthcare (PervasiveHealth '16). ISBN: 978-1-63190-051-8 